\title{
Windings Fault Detection and Prognosis in Electro-Mechanical Flight Control Actuators Operating in Active-Active Configuration
}

\author{
Andrea De Martin ${ }^{1}$, Giovanni Jacazio ${ }^{2}$, and George Vachtsevanos ${ }^{3}$ \\ ${ }^{1,2}$ Politecnico di Torino, Torino, 10129, Italy \\ andrea.demartin@polito.it \\ giovanni.jacazio@polito.it \\ ${ }^{3}$ Georgia Institute of Technology, Atlanta, GA, 30332, USA \\ gjv@ece.gatech.edu
}

\begin{abstract}
One of the most significant research trends in the last decades of the aeronautic industry is the effort to move towards the design and the production of "more electric aircraft". Within this framework, the application of the electrical technology to flight control systems has seen a progressive, although slow, increase: starting with the introduction of fly-by-wire and proceeding with the partial replacement of the traditional hydraulic/electro-hydraulic actuators with purely electro-mechanical ones. This evolution allowed to obtain more flexible solutions, reduced installation issues and enhanced aircraft control capability. Electro-Mechanical Actuators (EMAs) are however far from being a mature technology and still suffer from several safety issues, which can be partially limited by increasing the complexity of their design and hence their production costs. The development of a robust Prognostics and Health Management (PHM) system could provide a way to prevent the occurrence of a critical failure without resorting to complex device design. This paper deals with the first part of the study of a comprehensive PHM system for EMAs employed as primary flight control actuators; the peculiarities of the application are presented and discussed, while a novel approach, based on short pre-flight/post-flight health monitoring tests, is proposed. Turn-to-turn short in the electric motor windings is identified as the most common electrical degradation and a particle filtering framework for anomaly detection and prognosis featuring a self-tuning non-linear model is proposed. Features, anomaly detection and a prognostic algorithm are hence evaluated through state-of-the art performance metrics and their results discussed.
\end{abstract}

Andrea De Martin et al. This is an open-access article distributed under the terms of the Creative Commons Attribution 3.0 United States License, which permits unrestricted use, distribution, and reproduction in any medium, provided the original author and source are credited.

\section{INTRODUCTION}

Following the latest developments of the aviation industry, Electro-Mechanical Actuators (EMAs) are slowly replacing the traditional electro-hydraulic or hydraulic solution for fly-by-wire flight controls in military and civilian applications. EMAs allow for the elimination of leakage issues, simplify both installation and maintenance and provide weight-competitive solutions, hence resulting attractive for both suppliers and flight operators (Pratt, 2000). However, due to safety issues, their use as primary flight control actuators is still limited to experimental aircraft or UAVs (Jensen, Jenney \& Dawson, 2000), (Derrien, Tievs, Senegas \& Todeschi, 2011), (Roemer \& Tang, 2015), while they are more rapidly advancing in nonsafe critical applications such as flap/slats control surfaces (Christmann, Seemann \& Janker, 2010), (Recksieck, 2012). In order to overcome the afore-mentioned safety issues, one of the possible solutions is to build a robust Prognostics and Health Management (PHM) system able to rapidly detect the insurgence of one or more faults and to provide a sufficiently accurate estimate of the Remaining Useful Life (RUL) of the degraded component(s). Several research efforts can be found in the literature, addressing the electric motor (Nandi, Tolivat \& Li, 2005), (Brown, Georgoulas, Bole, Pei, Orchard, Tang, Saha, Saxena, \& Goebel, 2009), (Belmonte, Dalla Vedova \& Maggiore, 2015), mechanical components (Balaban, Saxena, Goebel, Watson, Bharadwaj \& Smith, 2009), (Balaban, Saxena, Narasimhan, Roychoudhury, Goebel \& Koopmans, 2010), (Lessmeier, Enge-Rosenblatt, Bayer \& Zimmes, 2014), (Van Der Linden, Dreyer \& Dorkel, 2016) and Electronic Power Unit (EPU) (Brown, Abbas, Ginart, Ali, Kalgren \& Vachtsevanos, 2010), (Li, Chen \& Vachtsevanos, 2014). Electro-mechanical actuators can be subjected to a large number of possible failure modes, involving both the hardware components, that is the focus of this research, and the control software. Bonnet and Soukup (1991) and as 
remarked in (Cruz \& Cardoso, 2002) and in (Nandi et al., 2005), the most common failures in the electric drives occur in the EPU, in the motor windings and in the motor bearings. Focusing on the winding failures, three major failure modes can be defined: turn-to-turn short, phase-tophase short and phase-to-ground short. Each of these failure modes is related to the degradation of the insulating coating, which prevents the contact between the turns and other conductive components. The most frequent winding failure mode is the turn-to-turn short, as assessed through FMECA analysis in (De Martin, Jacazio \& Vachtsevanos, 2016): its inception can be due to sudden, unpredictable occurrences, such as mechanical overloads and/or undesired overheating, but it is more commonly caused by the progressive degradation of the insulator due to heat and chemical aggression, while its progression may evolve into one of the other fault types (Nandi et al., 2005). The present work proposes a feasible solution to perform the early detection and the prognosis of the turn-to-turn fault for the EMAs governing a primary flight control surface for a regional transport aircraft. Introducing a PHM system for flightcontrol components comes with several challenges that are emphasized by the adoption of the electro-mechanical technology. The first one is the lack of available experimental data: EMAs are still rarely employed in flightcontrol systems, so that reliable, historical data are not available. Other issues are related to the limitations of the computational power embarked on the vehicle, that impose the choice of simple, computationally inexpensive features and fault detection algorithms to detect the health status of each monitored subsystem within the aircraft. Moreover, the unpredictable nature of the command imposed to the actuators and the presence of random loads due to gust and turbulence make it difficult to extract significant data during flight, while the number of sensor has to be kept to a minimum to avoid cost increases and reliability issues. Finally, both the monitored system and the degradation mode under study are heavily non-linear and affected by non-Gaussian noise; the prognostic algorithm must hence be able to mirror this behavior to limit the prediction uncertainty and maximize its accuracy.

\section{SYSTEM CONFIGURATION}

The system configuration considered for the analysis is depicted in Fig. 1. Two electro-mechanical actuators, each featuring a brushless electric motor supplied through its own EPU and a mechanical transmission composed by a satellite gearbox and a roller screw, move the flight control surface which equivalent translational mass is approximately $50 \mathrm{~kg}$. Each electric motor may convey up to $1 \mathrm{~kW}$ of power, while the overall transmission ratio of the mechanical organs is equal to $2.51 \mathrm{rad} / \mathrm{mm}$. Control of each actuator is performed by three nested regulation loops working on the electric motor currents, driving the shaft speed and the end-user linear position.

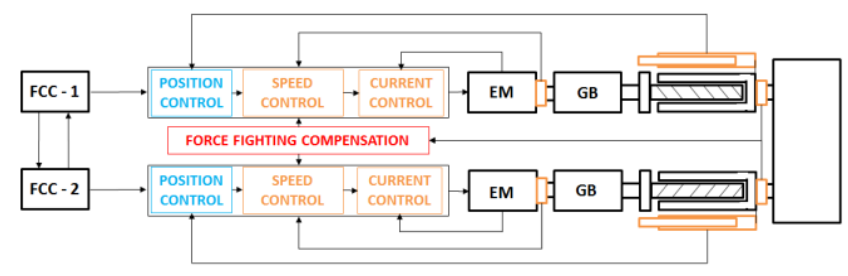

Figure 1. EMA Configuration

Feedback signals for each loop come from dedicated current sensors, a resolver positioned on the electric motor shaft and one or more linear position sensors (usually LVDTs) connected to the translating element of the power screw. Two inter-communicating Flight Control Computers (FCCs) provide the position command. The motion control is performed through an active/active strategy, which means that both devices are simultaneously actuated in position. This control scheme allows to obtain better dynamics response and/or to decrease the intensity of the current required by the motors, but suffers from force fighting occurrence. This dangerous phenomenon is due to the unavoidable small differences between the two actuators in terms of motor characteristics, friction parameters and clearances in the mechanical transmission: it usually shows up under low-load conditions, whenever one of the actuator begins to exert an action opposite to the commanded motion which is hence driven by the other electro-mechanical actuators. This may lead to windings overheating, further shortening the motor operative life. Force fighting can be compensated by motor current equalization or, if possible, by monitoring the force applied to each actuator using a proper set of transducers: their signal, filtered and sampled, can then be employed by a dedicated proportionalintegrative controller working either on the position or the speed loop (Wang, Maré \& Fu, 2012). For the studied system, force sensors are expected.

\section{SYSTEM MODEL}

To overcome the lack of historical data, we relied on a highfidelity dynamic model of the system prepared in the Matlab/Simulink environment. The model has been used to approximate the system behavior in a wide array of operating conditions to provide the basis for the PHM framework proposed in this paper. In this section, we introduce a brief overview of the mathematical model of the system and highlight the modifications used to describe the occurrence of a growing turn-to-turn short.

\subsection{Electric drive model}

The dynamic model of the electric drive is made of three main subsystems: the Electronic Control Unit (ECU), the Electronic Power Unit (EPU) and the brushless electric model. The model of the Electronic Power Unit makes use of a functional description of the PWM modulated inverter. It receives the commands from the ECU, which is 
responsible for the motor current control in the $d-q-0$ axis, and modulate the electric power exchanged with the motor. The $d-q$ axis control features PI regulators receiving as input the current command and the filtered current feedback subjected to Park's transformation. The output of the controllers is then transformed back to the three-phase system and used inside a PWM modulator, based on a triangular bipolar wave carrier that generates the digital control signal for each of the three commutation poles, namely $q_{1}, q_{2}$ and $q_{3}$.

Neglecting the power MOSFET dynamics, it is possible to compute the three motor phase voltages $v_{a}, v_{b}$ and $v_{c}$ as follows (Mohan, 2003):

$$
\left[\begin{array}{l}
v_{a} \\
v_{b} \\
v_{c}
\end{array}\right]=\eta_{E P U} \frac{V_{d c}}{2}\left[\begin{array}{ccc}
\frac{4}{3} & -\frac{2}{3} & -\frac{2}{3} \\
-\frac{2}{3} & \frac{4}{3} & -\frac{2}{3} \\
-\frac{2}{3} & -\frac{2}{3} & \frac{4}{3}
\end{array}\right]\left[\begin{array}{l}
q_{1} \\
q_{2} \\
q_{3}
\end{array}\right]
$$

Where $V_{d c}$ is the DC-link voltage used to supply the actuation system and $\eta_{E P U}$ is the EPU efficiency used to model the power dissipation due to resistive and commutation losses. The motor dynamics, under nominal conditions, are described through Eq. (2):

$$
\begin{gathered}
{\left[\begin{array}{l}
v_{a} \\
v_{b} \\
v_{c}
\end{array}\right]-\frac{d}{d t}\left[\begin{array}{l}
\lambda_{a} \\
\lambda_{b} \\
\lambda_{c}
\end{array}\right]=} \\
{\left[\begin{array}{ccc}
R_{a} & 0 & 0 \\
0 & R_{b} & 0 \\
0 & 0 & R_{c}
\end{array}\right]\left[\begin{array}{l}
i_{a} \\
i_{b} \\
i_{c}
\end{array}\right]+\frac{d}{d t}\left(\left[\begin{array}{lll}
L_{a a} & L_{a b} & L_{a c} \\
L_{a b} & L_{b b} & L_{b c} \\
L_{a c} & L_{b c} & L_{c c}
\end{array}\right]\left[\begin{array}{l}
i_{a} \\
i_{b} \\
i_{c}
\end{array}\right]\right)}
\end{gathered}
$$

Where $\lambda$ is the magnetic flux, which instantaneous value depends on its spatial distribution and on rotor angular position, while $R$ is the electric resistance and $L_{x y}$ is the mutual inductance between phases $x$ and $y$, as shown in the electric schematic reported in Fig. 2.

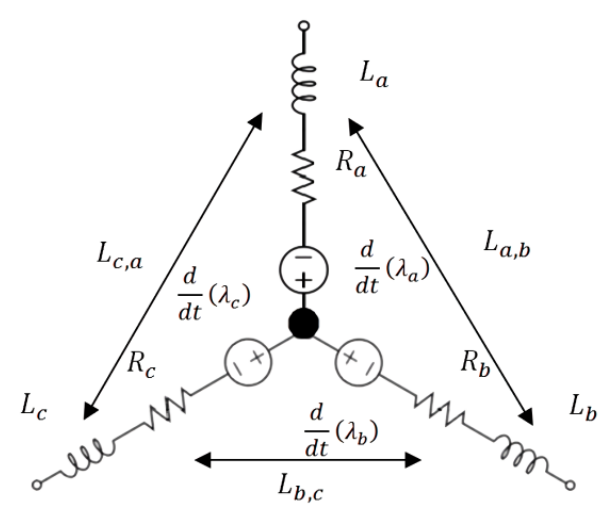

Figure 2. Brushless Motor Scheme
Given the number of pole pairs, $Z_{p}$, the electromagnetic torque can be obtained according to the following general relation (Hanselman, 2006):

$$
T_{e m}=\frac{3}{2} Z_{p}\left[\begin{array}{l}
\lambda_{a} \\
\lambda_{b} \\
\lambda_{c}
\end{array}\right] \wedge\left[\begin{array}{l}
i_{a} \\
i_{b} \\
i_{c}
\end{array}\right]
$$

It is finally possible to estimate the windings' thermal behavior:

$$
\left[\begin{array}{l}
i_{a} \\
i_{b} \\
i_{c}
\end{array}\right]^{T}\left[\begin{array}{ccc}
R_{a} & 0 & 0 \\
0 & R_{b} & 0 \\
0 & 0 & R_{c}
\end{array}\right]\left[\begin{array}{l}
i_{a} \\
i_{b} \\
i_{c}
\end{array}\right]-H_{t h} \vartheta_{t h}=C_{t h} \frac{d \vartheta_{t h}}{d t}
$$

Where $\vartheta_{t h}=T_{e m}-T_{\text {ext }}$ is the temperature difference between the motor windings and the external environment, while $H_{t h}$ and $C_{t h}$ are the thermal conductivity and the thermal capacity, respectively. According to (Brown, Georgoulas, Bole, Pei, Orchard, Tang, Saha, Saxena, Goebel \& Vachtsevanos, 2009), it is possible to model the presence of a turn-to-turn short in one of the motor phases by inserting the ratio $w_{f}$ in the electrical dynamic equation, thus modifying the motor circuit. This parameter may range between 0 and 1 and represents the ratio between the number of healthy windings over the total number of windings for the selected phase.

Equation (2) is then modified as follows:

$$
\begin{aligned}
& {\left[\begin{array}{l}
v_{a} \\
v_{b} \\
v_{c}
\end{array}\right]-\frac{d}{d t}\left[\begin{array}{l}
w_{f, a} \lambda_{a} \\
w_{f, b} \lambda_{b} \\
w_{f, c} \lambda_{c}
\end{array}\right]-\left[\begin{array}{ccc}
w_{f, a} R_{a} & 0 & 0 \\
0 & w_{f, b} R_{b} & 0 \\
0 & 0 & w_{f, c} R_{c}
\end{array}\right]\left[\begin{array}{l}
i_{a} \\
i_{b} \\
i_{c}
\end{array}\right]} \\
& =\frac{d}{d t}\left(\left[\begin{array}{lll}
w_{f, b} L_{a a} & w_{f, a b} L_{a b} & w_{f, a c} L_{a c} \\
w_{f, a b} L_{a b} & w_{f, b} L_{b b} & w_{f, b c} L_{b c} \\
w_{f, a c} L_{a c} & w_{f, b c} L_{b c} & w_{f, c} L_{c c}
\end{array}\right]\left[\begin{array}{l}
i_{a} \\
i_{b} \\
i_{c}
\end{array}\right]\right)
\end{aligned}
$$

Where $w_{f, i j}=\sqrt{w_{f, i} w_{f, j}}$. We address as "fault ratio" the reciprocal index $W_{f}=1-w_{f}$, which represents the number of faulted windings over the total number of windings for the phase under analysis. The effects of the fault progression on the phase currents are depicted in Fig. 3. The faulted winding signal tends to increase leading to current asymmetry and torque irregularity.
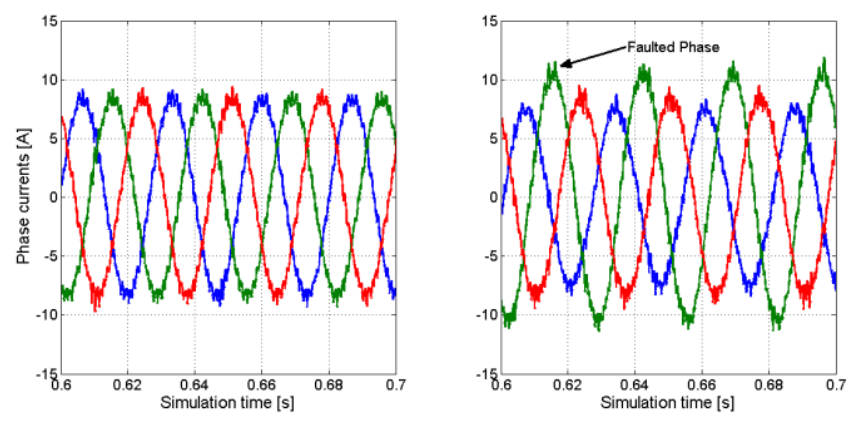

Figure 3. Effect of Turn-to-Turn Short on Phase Currents 
Turn-to-turn degradation can be triggered and driven by several causes, such as mechanical stresses, chemically aggressive environment, water ingress or excessive humidity (Nandi et al., 2005). However, the most significant and common origin is the insulator's thermal degradation (Brown et al., 2009). According to these authors, it is possible to describe the fault length $L$, expressed in [mm], as a function of the operating time $t$, the temperature of the windings, $T_{w}$, and the experimental coefficients $\alpha$ and $\beta$ through an elaboration of the Arrhenius law (Gokdere, Bogdanov, Chiu, Keller \& Vian, 2006):

$$
\left\{\begin{array}{l}
L=L_{0} e^{\gamma t} \\
\gamma=\beta e^{\alpha T_{W}}
\end{array}\right.
$$

Dividing the first expression of Eq. (6) by the critical length of the defect, it is possible to obtain a similar relation for the fault rate

$$
\left\{\begin{aligned}
W_{f} & =W_{f, 0} e^{\gamma t} \\
\gamma & =\beta e^{\alpha T_{W}}
\end{aligned}\right.
$$

Where $W_{f}=1-w_{f}$ and $W_{f, 0}$ are respectively the instantaneous and the initial fault ratios for the degraded phase.

\subsection{Mechanical transmission model}

The mechanical transmission is modelled, including the non-linear friction law and a customizable elasto-backlash, following the approach proposed in (Nordin, Gallic \& Gutman, 1997). Each gearbox is described through its dynamic equilibrium equation:

$$
T_{e m} \eta_{g b}-\frac{T_{r s}}{\tau}-T_{f r, g b}=J_{g b} \ddot{\vartheta}_{g b}
$$

Where $\tau$ is the transmission ratio, $\eta_{g b}$ is the meshing efficiency function of the operative temperature and of the gear dimensions; $J_{g b}$ is the moment of inertia and $T_{f r, g b}$ is the friction torque dependent on temperature and on the actuator speed: the friction law has been approximated through the non-linear Eq. (9) to avoid discontinuities:

$$
T_{f r, g b}=\min \left(T_{f r, g b s}, T_{f r, g b D}+\frac{1}{X_{F} \dot{\vartheta}_{g b}}\right)+c_{g b} \dot{\vartheta}_{g b}{ }^{2}
$$

Where $T_{f r, g b S}$ and $T_{f r, g b D}$ are the static and the sliding friction torques, $X_{F}$ is a customizable scaling factor and $c_{g b}$ is the speed dependent friction coefficient.

The torque transmitted to the actuator power screw, $T_{r s}$, depends on the relative position between the gearbox output shaft and the screw angular position as described by Eq. (10).

$$
\left\{\begin{array}{lr}
T_{r s}=0 & b_{\text {min }}<\Delta \vartheta<b_{\text {max }} \\
T_{r s}=k \Delta \vartheta+c \Delta \dot{\vartheta} & \Delta \vartheta \leq b_{\text {min }} \vee \Delta \vartheta \geq b_{\text {max }}
\end{array}\right.
$$

Where $k$ and $c$ are the stiffness and damping coefficients of the contact zone, while $b_{\min }$ and $b_{\max }$ determine the beginning and the end of the backlash. By selecting the proper combination of values for these parameters it is possible to simulate wear progression in the mechanical transmission.

Each power screw is modelled according to Eq. (11):

$$
T_{r s} \eta_{r s}-F_{s} \frac{p}{2 \pi}-T_{f r, r s}=J_{r s} \ddot{\vartheta}_{g b}
$$

Where $F_{s}$ is the linear force acting on the actuator; the friction torque is computed through Eq. (12).

$$
T_{f r, r s}=\min \left(T_{f r, r s S}, T_{f r, r s D}+\frac{1}{X_{F} \dot{\vartheta}_{r s}}\right)+c_{r s} \dot{\vartheta}_{g b}
$$

\subsection{Flight control surface model}

The aerodynamic surface is modelled according to the diagram shown in Fig. 4, while The dynamic equilibrium of this component can then be expressed through Eq. (13).

$$
\begin{gathered}
k_{s}\left(x_{1}-y\right)+k_{s}\left(x_{2}-y\right)+c_{s}\left(\dot{x}_{1}-\dot{y}\right) \\
+c_{S}\left(\dot{x}_{2}-\dot{y}\right)-F_{A}=m_{s} \ddot{y}
\end{gathered}
$$

Where $k_{s}$ and $c_{s}$ are the aerodynamic surface stiffness and damping factor, $F_{A}$ is the aerodynamic force acting on the actuators and $m_{s}$ the control surface equivalent mass.

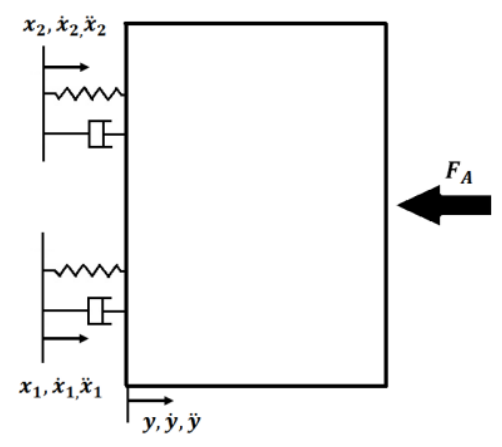

Figure 4. Control Surface Scheme

Where $k_{s}$ and $c_{s}$ are the aerodynamic surface stiffness and damping factor, $F_{A}$ is the aerodynamic force acting on the actuators and $m_{s}$ the control surface equivalent mass.

\section{HEALTH MONITORING STRATEGY}

To develop and evaluate a reliable prognostics and health management system it is fundamental to find one or more features or characteristic indicators from raw data able to comprehensively describe the health status of the monitored subsystem during its operating life.

For the turn-to-turn short fault, several possibilities are available in the literature (Nandi et al., 2005). Some of them make exclusive use of current analysis, while others involve a measure of the phase voltages. Voltage measurements are not available in the case under analysis; to avoid the 
introduction of additional sensors, current-based features are hence employed. We focused on simple, computationally inexpensive features to allow for on-board calculations.

Three features have been preliminary selected by combining in different ways the RMS of the three current signals as in Eq. (14).

$$
\left\{\begin{array}{l}
f_{i}^{1}=\frac{1}{3} \sum_{i=1}^{3} \mathrm{RMS}_{i} \\
f_{i}^{2}=\frac{1}{3} \sum_{i=1}^{3} \Delta \mathrm{RMS}(i) \\
f_{i}^{3}=\max (\Delta \mathrm{RMS})-\min (\Delta \mathrm{RMS})
\end{array}\right.
$$

Where $\mathrm{RMS}_{\mathrm{i}}$ and $\Delta \mathrm{RMS}_{\mathrm{i}}$ are respectively:

$$
\begin{gathered}
\mathrm{RMS}_{i}=\sqrt{\frac{1}{n_{s}} \sum_{j=1}^{n_{s}} i_{j}^{2}} \\
\Delta \mathrm{RMS}=\left[\begin{array}{l}
\left|\mathrm{RMS}_{1}-\mathrm{RMS}_{2}\right| \\
\left|\mathrm{RMS}_{1}-\mathrm{RMS}_{3}\right| \\
\left|\mathrm{RMS}_{2}-\mathrm{RMS}_{3}\right|
\end{array}\right]
\end{gathered}
$$

The current samples number is $n_{s}$, while each of their values is denoted as $i_{j}$. Addressing with $f_{i}$ a generic feature computed through post-processing of the current measure, the following dependence can be expressed as:

$$
f_{i}=f_{i}\left(x_{c}, \omega, v\right)
$$

Where $x_{c}$ is the system health status, $\omega$ is the measurement noise and $v$ the process noise. Ideally, the dependence of the health index over the two noise types should be null. The measurement noise can be reduced in practice by using properly-designed low-pass filters on the sensor signals and eventually post-processing the obtained data. The process noise is instead more difficult to handle and mainly depends on the nature of the feature, variability of the operating conditions, adopted control scheme and effects of external disturbances. The influence of the operating conditions on the current measurements can be easily understood by observing the simplified model reported in Eq. (5). As reported by Hanselman (2006), the temperature may have a significant influence on the motor parameters, while nonconstant speed conditions may cause a significant variation of the current measurements because of the backelectromotive force effect. The aerodynamic force eventually acting on the control surface and the overall friction torque sensed at the motor shaft may provide a major contribution to the process noise as well because of the presence of the closed-loop control system. For the same reasons, inertial load may have a critical effect as well. Primary flight controls lack of an established usage pattern during the flight; the number, duration and nature of the actuation associated with each mission are completely unpredictable a priori. In a similar way, the temperature profile experienced by the actuators can be extremely variable.

To overcome these issues and limit the process noise, Jacazio, Maggiore, Della Vedova and Sorli (2010) proposed the use of dedicated pre-flight/post-flight procedure for the Electro-Hydraulic Actuators. Following this approach, it is not only possible to limit the uncertainties due to environmental conditions, but also to stimulate the actuators with a predefined set of commands optimized to extract the required features and emphasize the effects of a fault occurrence. In this case, a slow ramp position command, with rate equal to $20 \%$ of the motor maximum speed, is imposed to the "monitored" actuator, where this low value is chosen to limit the influence of the back electromotive force over the measured current. The second "opposing" actuator is controlled in force, taking advantage of the force sensors already present for the force-fighting compensation. Through this approach it is possible to reduce the influence of friction over the force exerted by the second actuator. The commanded force is again a ramp saturated at $40 \%$ of the nominal value that is high enough to enhance the current analysis while remaining far enough from saturation conditions, which would make the current measurements useless. The influence of random gust loads during the test performed on the airport track can be considered negligible and can be eventually cancelled through the implementation of a simple force compensation making use of the available force sensors. The proposed pre-flight test is 1-second-long, while the acquisition is performed over the last $0.5 \mathrm{~s}$. Once completed, the operation is repeated inverting the roles of the two actuators.

\subsection{Feature selection}

Three feature candidates have been proposed. Each feature behavior has been preliminarily investigated by performing several simulations while considering a large number of external disturbances and parameters variations. In particular, the environmental temperature has been randomly chosen from a uniform distribution ranging between $-40^{\circ} \mathrm{C}$ and $40^{\circ} \mathrm{C}$, while each coefficient used to determine the friction behavior and its dependence on the temperature is subjected to a variation determined by a normal distribution with standard deviation equal to $40 \%$ of their nominal value. A similar approach has been used for the electric motor parameters, whereas the standard deviation has been set to $1 \%$. Finally, the external load has been simulated as a step signal occurring in a random moment during the pre-flight test; its intensity has been chosen each time from a normal distribution set with $0 \mathrm{~N}$ mean and standard deviation equal to $20 \%$ of the maximum value. Simulations have been performed using the Matlab/Simulink implementation of the dynamic model presented in Section 3; while acquiring current signals, the fault ratio $W_{f}$ has been slowly increased from 0 up to 0.7 . 


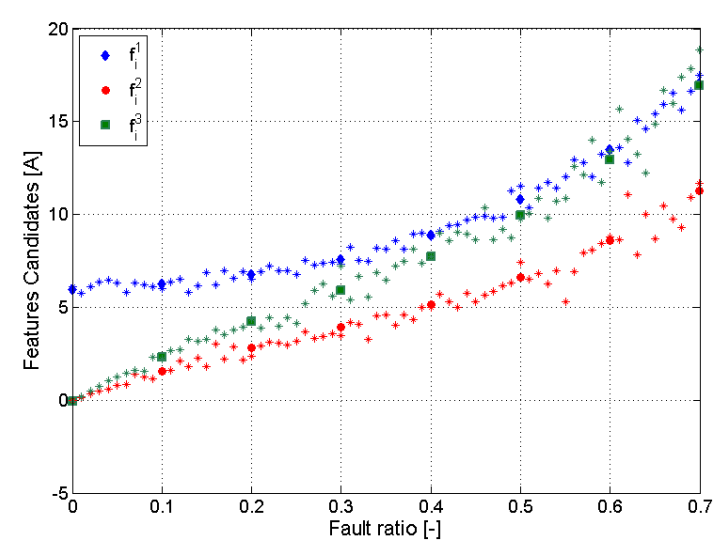

Figure 5. Feature Candidates' Behaviour

Results of the feature candidates' behavior are reported in Fig. 5. The performance of the selected features is analyzed through two dedicated metrics: the accuracy measure and the precision measure. Accuracy is defined as the linear correlation between the generic feature $f_{i}$ and the fault ratio $W_{f}$. Precision is instead computed as the relative mean error associated with the functions used to interpolate the features' behavior. Feature candidate $f_{i}^{l}$ has shown overall better performance, averaging an accuracy measure equal to 0.932 and a precision metrics of $3.94 \%$. The other options, $f_{i}^{2}$ and $f_{i}^{3}$, exhibit worse accuracy $(0.925$ and 0.921$)$ and worse precision (over $9 \%$ in both cases).

\section{ANOMALY DETECTION}

A generic Prognostic and Health Management framework is depicted in Fig. 6. The first stage of any health monitoring operation is always the fault diagnosis: this includes three main tasks, namely, fault detection, fault isolation and finally fault severity (or identification). It implies that the system must be able to detect the occurrence of one or more faults or incipient failures, address the targeted component(s) and finally assess the fault severity as for the specified performed measures (Vachtsevanos, Lewis, Roemer, Hess \& Wu, 2006). In this process, the knowledge of the degradation process obtained through off-line studies is fundamental to define the diagnosis method and eventually to perform the diagnosis itself. Since this paper deals with a single-fault scenario, only the fault detection is addressed herein. Once a fault has been declared, data are analyzed by the prognostic algorithm(s) that estimates the Remaining Useful Life (RUL) of the damaged component. In order to set up the basis for a reliable prognostic algorithm, fault diagnosis must be able to minimize the false positive and false negative errors while providing early defect detection. The adopted fault diagnosis method provides a diagnostic result at a customer specified accuracy (confidence) with a given false alarm rate. Two approaches are evaluated to perform the anomaly detection: a purely data-driven method and a particle-filtering framework. Both systems are detailed and results compared.

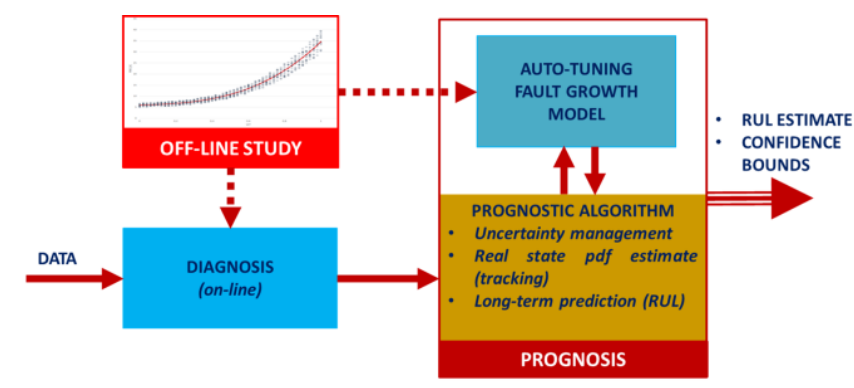

Figure 6. General scheme for PHM framework

\subsection{Operational scenario}

As described by Eqs. (6-7), the evolution in time of the Turn-to-Turn Short fault is mainly dependent on the temperature of the windings. This means that the external environmental temperature, the external load and the local thermal exchange conditions govern the fault progression. Each of these factors is in turn dependent on the aircraft class, take-off/landing areas and weather conditions. For this work, a generic regional transport aircraft, a vehicle moving passengers or goods over mid-range routes, is considered. The average loads and the duration of each flight phase (pre/post flight, take-off, cruise, landing) has been defined as the sum of a constant value and a random variable inserted to simulate the effects of different weather conditions and unpredicted delays in the operating schedule. The average flight duration has been set equal to 1.5 hours. Atmospheric temperature during take-off and landing has been assumed to be linearly varying between the ground value, chosen randomly between $-40^{\circ} \mathrm{C}$ and $+40^{\circ} \mathrm{C}$, and $54^{\circ} \mathrm{C}$ during cruise (Phillips, 2004). Those considerations have been used in a simulation framework making use of the system model and the feature behavior described in Fig. 7. During each simulation cycle, the duration $t_{f s}$ of each flight segment is stored, while the average temperature of the windings is evaluated through Eq. (4); this value is then used to evaluate the fault ratio $W_{f}$. and to compute its evolution over the entire mission by iterating Eq. (7) for each flight segment. In order to ensure the fault occurrence, the initial instant $W_{f o}$ is non zero and is set to a value of $10^{-4}$.

\subsection{Anomaly detection through data-driven approach}

The first approach presented in this paper is a purely datadriven one (Vachtsevanos et al., 2006). A baseline representing the feature behavior under healthy conditions is built upon the first 100 data points and an automatic, customizable threshold is set to a value covering $95 \%$ of the baseline probability distribution. Simulation data are then streamed in and compared to the initial baseline; the anomaly detection flag is raised when the new distribution differs from the baseline by a customizable confidence level equal to or greater than $95 \%$. This approach has been applied to 20 data sets with different environmental conditions. The selected feature $f_{i}^{l}$ shows an average fault 
ratio at detection of $12.71 \%$. An example of the behavior of the data-driven method for an extremely slow degradation is reported in Fig. 7. As expected, the detection confidence tends to oscillate for a low value of the fault ratio, hence increasing in a consistent way once the degradation begins to accelerate. The fault is declared with a detection confidence equal to $95 \%$. Hence, the Type I error, defined by the user, is fixed at $5 \%$ while the Type II error under these conditions has a value equal to $5 \%$.
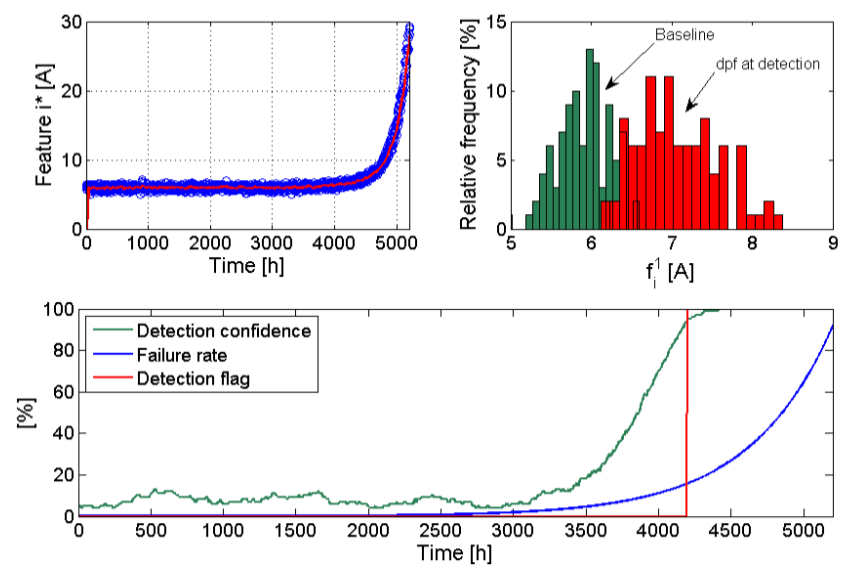

Figure 7. Output of the fault detection framework

\subsection{Anomaly detection through particle-filtering framework}

Particle filtering is a powerful Bayesian estimator that allows to approximate non-linear dynamic processes affected by non-Gaussian noise and is hence well suitable for the examined problem; its characteristics can be exploited to perform anomaly detection making use of a reduced particle population to represent the state probability density function (Orchard, 2007). This framework provides an estimate of the probability masses associated with each fault mode, as well as a pdf estimate for meaningful physical variables in the system. Once this information is available within the diagnostic module, it is conveniently processed to generate proper fault alarms and to inform about the statistical confidence of the detection routine. Customer specifications are translated into acceptable margins for the type I and II errors in the detection routine. The algorithm itself will indicate when the type II error (false negatives) has decreased to the desired level. The particle filtering approach is based upon the following general nonlinear dynamic state model

$$
\left\{\begin{array}{l}
x_{d}(t+1)=f_{b}\left(x_{d}(t), n(t)\right) \\
x_{c}(t+1)=f_{t}\left(x_{d}(t), x_{c}(t), \omega(t)\right) \\
f_{p}(t)=h_{t}\left(x_{d}(t), x_{c}(t), v(t)\right)
\end{array}\right.
$$

Where $f_{b}, f_{t}$ and $h_{t}$ are non-linear mappings, $x_{d}$ is a collection of Boolean states associated with the presence of a particular operating condition in the system (normal operation, fault type $\# 1$, $\# 2$, etc.), $x_{c}$ is a set of continuousvalued states that describe the evolution of the system given those operating conditions, $f_{p}$ is a feature measurement, $\omega$ and $v$ are non-Gaussian distributions that characterize the process and feature noise signals, respectively. The function $h_{t}$ is a mapping between the feature value, $f_{p}(t)$, and the fault state $x_{c}(t)$. At any given instant of time, this framework provides estimates of fault detection only when customer specified confidence and false alarm metrics are met. Furthermore, pdf estimates for the system continuousvalued states may be used as initial conditions in failure prognostic routines resulting in a swift transition between the two modules (FDI and prognosis). This approach has been employed only for the selected feature $f_{i}^{l}$. The $f_{t}$ expression is derived from theory and is linked to Eq. (13). The $h_{t}$ mapping has been investigated by making use of the symbolic regression method based on a genetic algorithm provided by (Schmidt, Lipson, 2009), hence obtaining the polynomial expression reported in Eq. (19)

$$
f_{i}^{1}=a w_{f}^{3}+b w_{f}^{2}+c w_{f}+d
$$

By exploiting the particle filtering framework, it is possible to obtain appreciable performance benefits for the diagnosis procedure; the average fault ratio associated with the detection time, computed over the 20 data sets, is in fact reduced to $10.48 \%$, when using the same percentage thresholds applied to the purely data driven case. However, due to the reliance on a simplified degradation model, the Type I and Type II errors tend to increase up to $8.1 \%$ and $6.7 \%$, respectively. As such, the simpler data-driven approach has been selected and implemented in the prognostic and health management framework.

\section{PARTICLE FILTERING FOR PROGNOSIS}

The prognostic framework takes advantage of a nonlinear process (fault / degradation) model, a Bayesian estimation method using particle filtering and real-time measurements (Vachtsevanos, et al., 2006). Prognosis is achieved by performing two sequential steps, prediction and filtering (Orchard, 2007), (Orchard \& Vachtsevanos, 2009). Prediction uses both the knowledge of the previous state estimate and the process model to generate the a priori state pdf estimate for the next time instant,

$$
\begin{gathered}
p\left(x_{0: t} \mid y_{1: t-1}\right) \\
=\int p\left(x_{t} \mid y_{t-1}\right) p\left(x_{0: t-1} \mid y_{1: t-1}\right) d x_{0: t-1}
\end{gathered}
$$

Unfortunately, this expression does not have an analytical solution in most cases (Roemer, Byngton, Kackprszynski, Vachtsevanos \& Goebel, 2011). Instead, Sequential Monte Carlo (SMC) algorithms (Arumpalan, Maskell, Gordon \& Clapp, 2002), or particle filters, are used to numerically solve this equation in real-time with efficient sampling strategies. The workflow of the employed particle filtering method is reported in Fig. 8. 


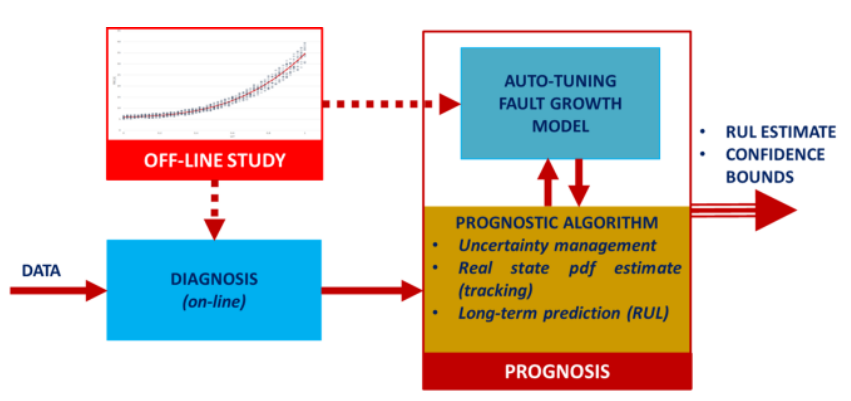

Figure 8. The Prognostic Framework

The particle filter approximates the state pdf using samples or "particles" having associated discrete probability masses ("weights") as,

$$
p\left(x_{t} \mid y_{1: t}\right) \approx \widetilde{w}_{t}\left(x_{0: t}^{i}\right) \delta\left(x_{0: t}-x_{0: t}^{i}\right) d x_{0: t-1}
$$

where $x_{0: t}^{i}$ is the state trajectory and $y_{1: t}$ are the measurements up to time $t$. According to Orchard (2007), the simplest implementation of this algorithm, the Sequential Importance Re-sampling (SIR) particle filter, updates the weights using the likelihood of $y_{t}$ as

$$
w_{t}=w_{t-1} p\left(y_{t} \mid x_{t}\right)
$$

Long-term predictions are used to estimate the probability of failure in a system given a hazard zone that is defined via a probability density function with lower and upper bounds for the domain of the random variable, denoted as $H_{l b}$ and $H_{u p}$, respectively. The probability of failure at any future time instant is estimated by combining both the weights $w^{(i)}{ }_{t+k}$ of predicted trajectories and specifications for the hazard zone through the application of the Law of Total Probabilities. The resulting RUL pdf, where $t_{R U L}$ refers to RUL, provides the basis for the generation of confidence intervals and expectations for prognosis,

$$
\hat{p}_{t_{R U L}}=\sum_{i=1}^{n} p\left(\text { Failure } \mid X=\hat{x}_{t_{R U L}}^{(i)}, H_{l b}, H_{u p}\right)
$$

As shown in Fig. 9, this approach makes use of degradation models that are tuned or their parameters adjusted to compute the current a-priori state of the system, $p\left(x_{t} \mid y_{1: t-1}\right)$, and to perform the iterative calculation that leads to the long term prediction $p\left(x_{t+k} \mid y_{1: t}\right)$. Auto-tuned models are required to describe and follow changes in the degradation process and to describe, in the best possible way, the process and measurement noise. So, in order to match the model behavior to the real system, as closely as possible, one time-dependent tunable model is used, following an approach similar to that described in ( $\mathrm{Li} \&$ Vachtsevanos, 2015). Degradation detection and prediction algorithms rely on data, a model of the degradation process and an estimation method that, given the current state of the system, predicts its evolution over the next time step. Such models are typically based on first principles while others are built on the basis of data. We exploit in this effort a modeling framework called Symbolic Regression. Symbolic Regression searches the space of mathematical expressions to find the model that best fits a given dataset, both in terms of accuracy and simplicity. No particular model is provided as a starting point to the algorithm. Instead, initial expressions are formed by randomly combining mathematical building blocks such as mathematical operators, analytic functions, constants, and state variables. (Usually, a subset of these primitives will be specified by the operator, but that is not a requirement of the technique.) New equations are then formed by combining previous ones, using genetic programming. In linear regression, the dependent variable is a linear combination of the parameters (but need not be linear in the independent variables). Nonlinear Symbolic Regression and other regression techniques incorporating uncertainty are based on similar principles. We take advantage of the Symbolic Regression tool provided in (Schmidt \& Lipson, 2009) to represent an expression linking the selected feature $f_{i}^{l}$ to operating time:

$$
f_{i}^{1}(t)=A+B t^{2}+C e^{D t}
$$

The model parameters can be tuned on-line as data is streaming in, following an iterative procedure featuring a recursive least square algorithm over a moving time window involving the last 100 data points. Fitting quality and most significantly the time required to converge to the real data sequence depend on the initial guess for the model parameters and on the size of the moving window. An example of model tuning for a slow degradation is shown in Fig. 9.

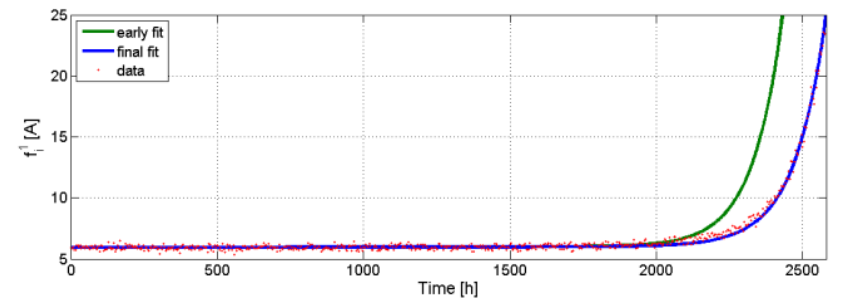

Figure 9. Feature Model - Fitting Results

\subsection{Remaining Useful Life prediction}

The particle filter algorithm estimates the Remaining Useful Life (RUL) by iterating its prediction step until the $95 \%$ of the 500 used particles overcome a user-defined threshold. This threshold has been set at $24 \mathrm{~A}$, which is more than four times the average healthy value and is associated with a mean fault ratio equal to 0.6 . The prediction can be performed after each mission of the aircraft under study. Addressing as $t_{E O L}$ the estimated end of lifetime for the electric motor and as $t_{p}$ the prediction time, that is the instant at which the RUL prediction is performed, it is possible to compute the RUL as (Vachtsevanos et al., 2006):

$$
\mathrm{RUL}=t_{E O L}-t_{P}
$$



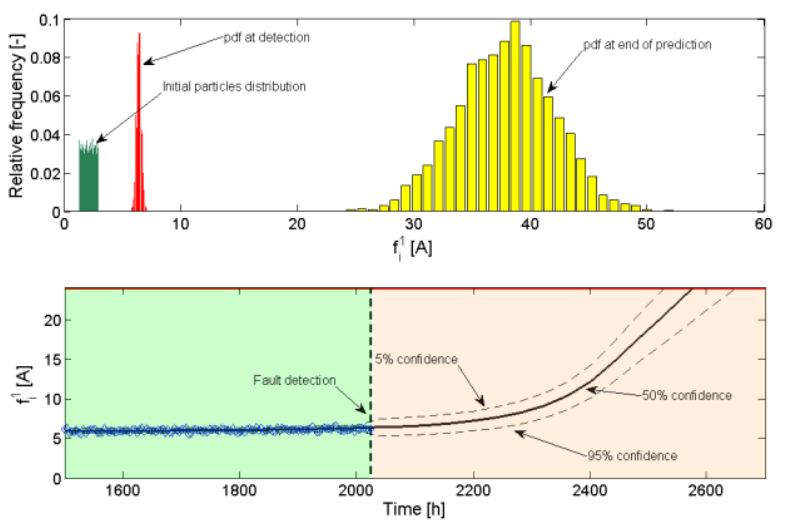

Figure 10. Prognosis results

An example of the analysis performed over a slow degradation is depicted in Fig. 10. Particles have been initially defined as a uniform distribution within the $[0,1]$ interval. The algorithm tracks the feature trend in a few iterations and approximates the data distribution. The RUL is often computed with reference to the $50 \%$ confidence trajectory of the particle filter. Other user-defined limits, such as the 5\% confidence and the $95 \%$ confidence trajectories, can be used to define the time to perform early or late maintenance, respectively.

\subsection{Prognosis performance}

The performances of the prognostic algorithm are evaluated through the metrics proposed by Saxena, Celaya, Balaban, Goebel, Sasha and Schwabacher (2008), and frequently adopted in the literature: the relative accuracy, RA, and the cumulative relative accuracy CRA. The $\alpha-\lambda$ analysis has been performed as well in order to assess in the best possible way the system performance.

The $\alpha-\lambda$ analysis is used to visually display and verify that the RUL prediction remains (or not) inside the accuracy limit for a generic time instant $t=t_{D}+\lambda\left(t_{E O L}-t_{D}\right)$, where $t_{D}$ is the first prediction time and $\lambda$ a scale factor ranging between 0 and 1. In Fig. 11 and in Fig. 12, the RUL is normalized with respect to the real RUL at fault detection. Observing them, it is possible to highlight how the system performance depends on the degradation speed, namely the slower the degradation the more stable and precise is the algorithm output. The relative accuracy is a measure of the algorithm's capability to estimate precisely the RUL at a generic time instant $t$.

$$
R A=1-\frac{\left|\mathrm{RUL}_{r}-\mathrm{RUL}\right|}{\mathrm{RUL}_{r}}
$$

Where $R U L_{r}$ is the ground-truth value for the remaining useful life. The cumulative value of RA, introduced to better evaluate the system accuracy toward the end of life of the component, can be defined as:

$$
C R A=\frac{1}{\sum_{i=1}^{l} w_{i}} \sum_{i=1}^{l} w_{i} R A_{i}
$$

Where each weight $w_{i}$ has been assumed to be equal to the corresponding $\lambda_{i}$. Each index has been evaluated over the 20 data sets, leading to the results depicted in Fig. 13. The mean value of the RA is equal to $86.55 \%$, while the average CRA at the end of prediction is $87.84 \%$. In Fig. 14, the CRA values for each data set are reported as well as the overall minimum, maximum and average value, for each value of $\lambda$. Prognosis performance is stable over every considered data set, consistently achieving CRA values higher than $80 \%$ for $\lambda$ higher than 0.4 .

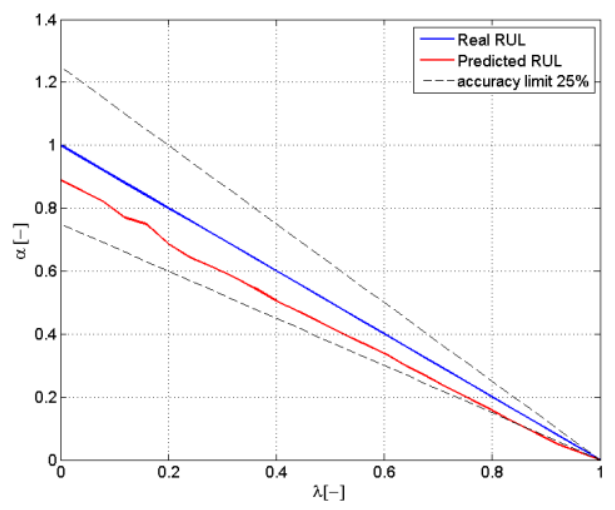

Figure 11. $\alpha-\lambda$ Analysis for a 600 hours degradations

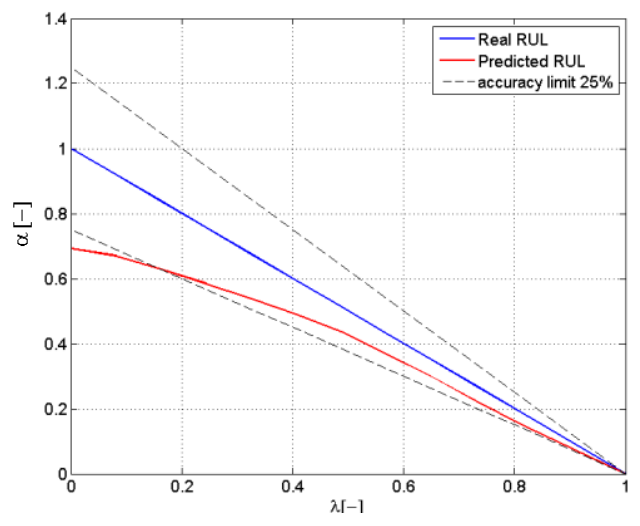

Figure 12. $\alpha-\lambda$ Analysis for a 315 hours degradations

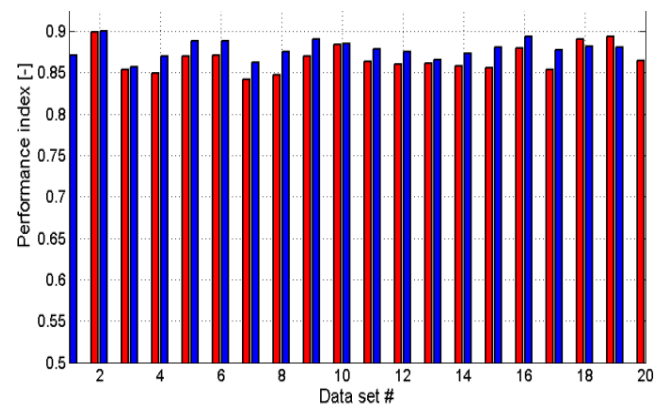

Figure 13. CRA at End of Prediction and Mean RA for Data Set \#1-20 


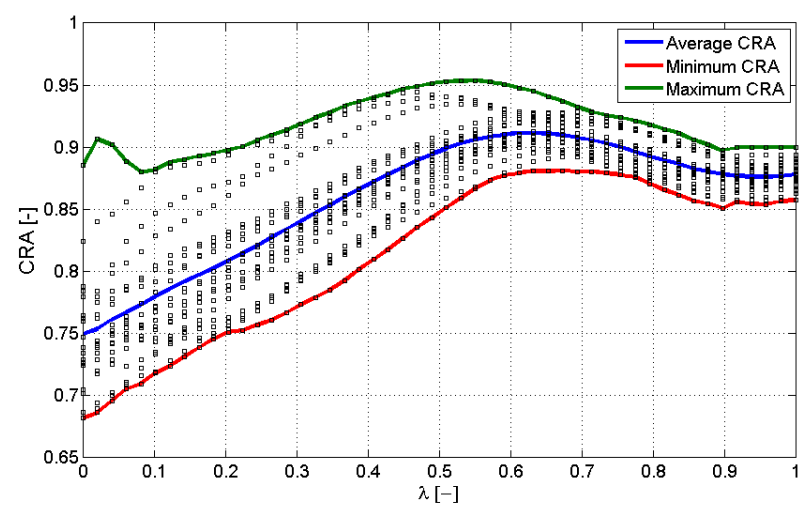

Figure 14. CRA at Several Prediction Times

\section{SYSTEM LIMITATIONS AND FURTHER DEVELOPMENTS}

The main limitation of the proposed PHM system is related to its performance degradation for faults that evolve very quickly compared to the framework convergence rate. This issue is due to the use of an auto-tuning algorithm for the non-linear degradation model employed in the particle filter. This routine makes use of the last 30 values of the selected feature, each obtained after a pre-flight or post-flight check; hence the tuning algorithm might be too slow to converge to the optimal solution, leading to inaccurate RUL estimates. This issue cannot be solved easily while keeping the pre/post flight checks strategy, while it could be overcome through the use of the in-flight data; it is clear that a change in the PHM strategy will necessarily require the study of other features and eventually the use of some additional sensors.

Another limitation is represented by the system behavior in multi-fault scenarios. So far the system behavior has been studied under the hypothesis of single-fault occurrence, that is, no other faults have been considered in the two EMAs. Even if we can assume that other systems will be used to detect the presence of other degradation processes, it is still important to assess the robustness of the presented methodology in multi-fault scenarios. A few cases are considered: power loss in the supply line, magnets degradation in one of the electric motors and a severe mechanical efficiency loss in one of the actuators. Motors can experience an electric power loss due to multiple causes such as issues within the batteries, a fault in the power bus or the occurrence of a short in the capacitive DC-link of the power converter. Although causing significant issues during flight, the occurrence of these faulty situations is not harmful to the pre/post flight checks provided that the supplied power is still sufficient to perform the required movement. The degradation of the motors' permanent magnets could instead affect the pre/post flight checks in a more significant way. Thanks to the presence of the force sensors its occurrence in the "opposing" actuator is not significant as long as the actuator is able to provide the required $40 \%$ of the nominal load.
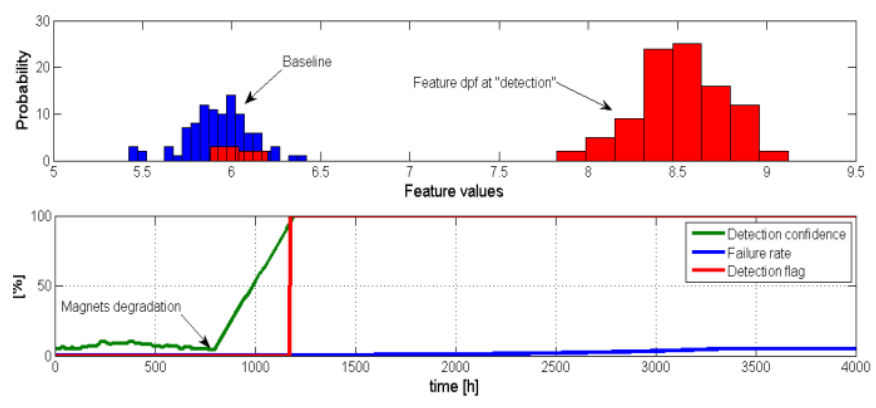

Figure 15. Example of a false positive error with a $30 \%$ degradation of the rotor magnets

On the other hand, its occurrence in the "monitored" actuator may raise false-positive flags: as shown in Fig. 15, the motor currents tend to increase to compensate for the torque loss hence causing a deviation in the feature normal behavior that, consequently, may lead to erroneous results of the PHM framework. A similar effect can be observed for a significant decrease in the mechanical transmission efficiency due to the occurrence of one or more mechanical defects, such as yielding of the ballscrew return channel, loss of lubricant or deformation of one of the rolling elements. To limit the influence of these disturbances, the use of a new feature, $f_{i}$, that makes use of additional voltage sensors, is proposed. This novel feature is defined as:

$$
f_{i}=\operatorname{std}\left(\frac{\operatorname{RMS}\left[i_{a, b, c}-\hat{\imath}_{a, b, c}\right]}{\operatorname{RMS}\left[V_{a, b, c}\right]+V_{o f f}}\right)
$$

Where $\hat{\imath}_{a, b, c}$ are the theoretical phase currents, $V_{a, b, c}$ are the phase voltages and $V_{\text {off }}$ a constant off-set added to avoid division by zero and numerical issues when the measured quantities are near the minimum quantization error. The theoretical currents are obtained by applying the Park antitransform to the $d-q-0$ axis quantities, computed by the motor control electronics, while neglecting the common node component.

The behavior of this novel feature with respect to the fault ratio is reported in Fig. 16. Repeating the procedure performed over the previously selected feature, it is observed that even the new one follows a time-dependent law of the type reported in Eq. (24).
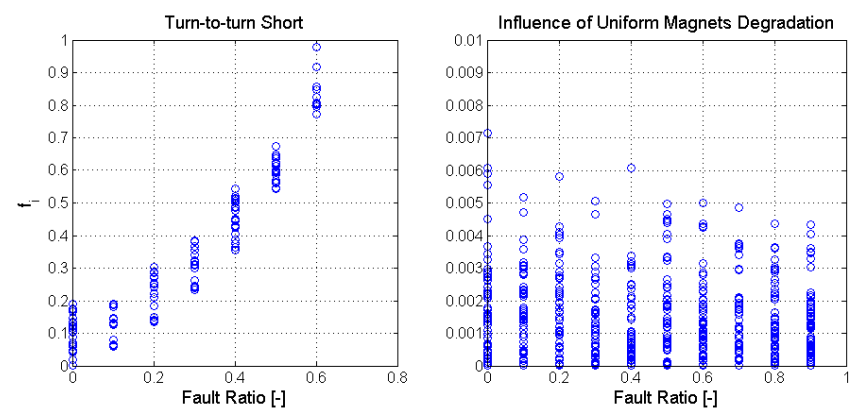

Figure 16. New feature behaviour 
Using the new feature in the proposed scheme for the datadriven fault detection and particle filter prognosis gives results similar to those obtained with previously selected feature, when considering single-fault scenarios. On the same 20 data sets, the average mean fault ratio at detection is $11.75 \%$; the mean RA is equal to $87.91 \%$ while the mean CRA at the end of prediction is $93.1 \%$. However, results are much more robust when considering the presence of other degradations; as shown in Fig. 16, the influence of uniform degradation in the rotors' magnets on the new feature is extremely limited, since it remains inside the process noise determined in its absence. An example of the framework's behaviour with the new feature is reported in Figs. 17 and 18.
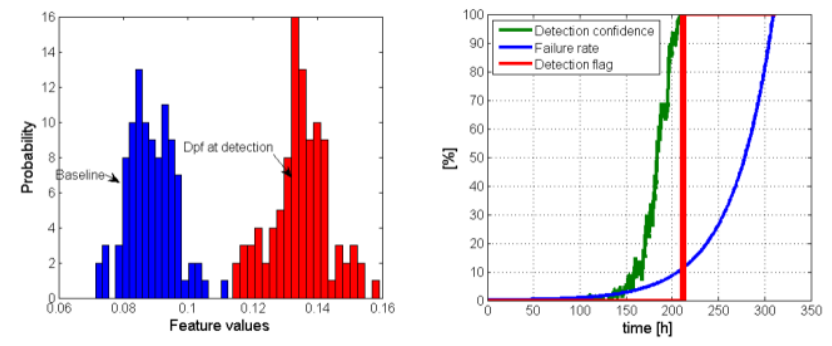

Figure 17. Fault detection
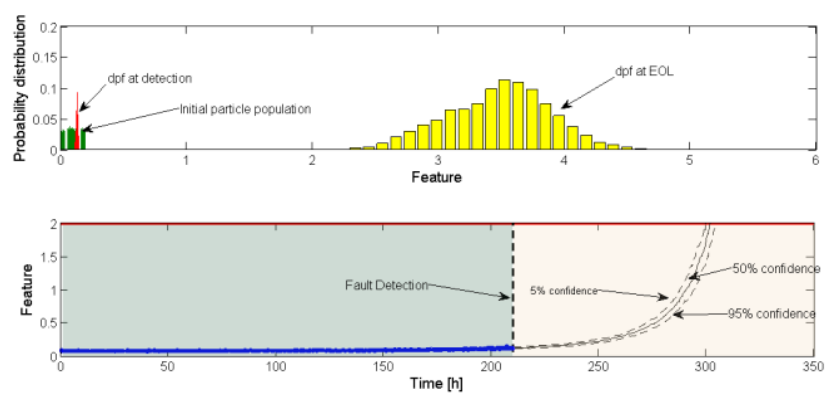

Figure 18. Output of the prognostic framework

\subsection{Validation plans}

Although we did not provide any experimental results to support the proposed framework, a few indications regarding its validation plan can be reported. EMAs are still rarely used for flight control system; as such, historical data are lacking. The validation of the proposed PHM system will have hence to rely on laboratory tests. The test bench will feature the two parallel EMAs connected to the equivalent translational mass through elastic joints to simulate the mechanical properties of the aerodynamic surface. A third actuator controlled in force may be added to simulate the effect of the wind. To validate the PHM algorithm we need two study the behavior of the selected feature in response to the fault inception and how the fault propagates in time. The first study can be pursued by inserting an external variable resistance in parallel with one of the motor's phases to mimic the fault inception and propagation without damaging its windings. Data would be obtained by progressively decreasing the value of the parallel resistance and performing the command sequence described in Section 4 under varying operating temperature. This approach has the advantage of being non-destructive, but lacks of any information regarding the temporal scale of the fault-to-failure process, which is critical for the validation of the prognostic algorithm. To pursue this objective, the only viable solution is to physically seed the fault and study the motor behavior during several flight simulations on the test bench or, preferably, on a dedicated iron-bird.

\section{Conclusions}

The turn-to-turn short has been identified as the most probable fault for brushless electric motors employed in flight control actuators. The physics of its inception and growth have been introduced and the issues related to its early detection in widely varying environmental conditions have been highlighted. In order to solve these issues, a novel set of pre-flight commands able to excite the system while enhancing the fault detectability has been proposed. Hence, a high-fidelity model of the system has been presented and used to supply to the lack of historical data. The macroscopic effects of the fault inception and the fault growth have been studied and a feature representative of the system health status has been chosen among a few candidates. This feature, based on simple current measurements, has been used to evaluate two possible anomaly detection methods: one following a data-driven approach and the other employing a particle filter algorithm. The first method, while slower in performing the fault detection, has proven to be more reliable and has as such been selected. On this basis, a particle filtering framework, based on auto-tuning the non-linear degradation model, has been developed for the prognosis of the turn-to-turn short failure mode. Performance has been evaluated through stateof-the-art metrics in single fault scenarios. Considering a few multi-faults scenarios, weakness in the system robustness has emerged; a new feature, based on the use of additional voltage sensors, has been hence introduced and its usage justified. The use of pre/post flight checks to limit data dispersion appears to be promising in performing the fault detection, tracking and prognosis for slow degradation patterns. However, the limited amount of data available, (only two measurements for each mission), makes this method less effective for fast degradation processes.

\section{REFERENCES}

Arulampalam, S., Maskell, S., Gordon, \& Clapp N. J. (2002). A Tutorial on Particle Filters for On-line Nonlinear/Non-Gaussian Bayesian Tracking. IEEE Trans. on Signal Processing, vol. 50 (2), pp. 174-188.

Balaban, E., Saxena, A., Goebel, K., Byington, C.S., Watson, M., Bharadwaj, S., \& Smith M. (2009). 
Experimental data collection and modeling for nominal and fault conditions on electro-mechanical actuators. Annual Conference of Prognostics and Health Management Society, September 27-October 1, San Diego, CA, USA.

Balaban, E., Saxena, A., Narasimhan, S., Roychoudhury, I., Gobel, K.F., \& Koopmans M. (2010). Airborne electromechanical actuator test stand for development of prognostic health management systems. Annual Conference of the Prognostics and Health Management Society, October 10-16, Portland, OR, USA.

Belmonte, D., Dalla Vedova, M.D.L., \& Maggiore, P. (2015). New prognostic method based on spectral analysis techniques dealing with motor static eccentricity for aerospace electromechanical actuators. WSEAS Transactions on Systems, vol. 14.

Bonnett, A.K., \& Soukup, G.C. (1991). Cause and analysis of stator and rotor failures in 3-phase squirrel cage induction motors. Pulp and Paper Industry Technical Conference, 3-7 June, Montreal, Quebec, Canada.

Brown, D., Abbas, M., Ginart, A., Ali, I., Kalgren, P., \& Vachtsevanos G. (2010). Turn-off time as a precursor for gate bipolar transistor latch-up faults in electric motor drives. Annual Conference of the Prognostics and Health Management Society, October 10-16, Portland, OR.

Brown, D.W., Georgoulas, G., Bole, B., Pei, H.L., Orchard, M., Tang, L., Saha, B., Saxena, A., Goebel, K., \& Vachtsevanos G. (2009). Prognostics enhanced reconfigurable control of electro-mechanical actuators. Annual Conference of the Prognostics and Health Management Society, September 27-October 1, San Diego, CA.

Christmann, M., Seemann, S., \& Janker, P. (2010). Innovative approaches to electromechanical flight control actuators and system. Recent Advances in Aerospace Actuation Systems and Components, May 57 Touluse, France.

Cruz, S.M.A., \& Cardoso, A.J.M. (2002). Stator winding fault diagnosis in three-phase synchronous and asynchronous motors, by the extended Park's vector approach, IEEE Transactions on Industry, vol. 37 (5).

De Martin, A., Jacazio, G., \& Vachtsevanos, G. (2016). Anomaly detection and prognosis for primary flight control EMAs, $3^{\text {rd }}$ European Conference of the Prognostics and Healh Management Society, July 5-8, Bilbao, Spain.

Derrien, J., Tieys, P., Senegas, D., \& Todeschi, M. (2011). EMA Aileron COVADIS development, SAE Technical Paper 2011-01-2729.

Gokdere, L. U. , Bogdanov, A., Chiu, S. L., Keller, K. J. \& Vian, J. (2006). Adaptive control of lifetime. IEEE Aerospace Conference, March 4-11, Big Sky, MT, USA.

Hanselman, D. (2006). Brushless Permanent Magnet Design. Magna Physics.
He, C., Li, J., \& Vachtsevanos, G. (2015). Prognostics and health management of an automated machining process, Mathematical Problems in Engineering, vol. 2015.

Jacazio, G., Maggiore, P., Della Vedova, M., \& Sorli, M. (2010). Identification of precursors of servovalves failures for implementation of an effective prognostics. Proceedings of the $4^{\text {th }}$ International Conference on Recent Advances in Aerospace Actuation Systems and Components, May 5-7, Toulouse, France.

Jensen, S.C., Jenney, G.D., \& Dawson, D. (2000). Flight test experience with an electromechanical actuator on the F-18 system research aircraft. Proceedings of the 19th DASC 1, October 7-13, Philadelphia, PA, USA.

Lessmeier, C., Enge-Rosenblatt, O., Bayer, C., \& Zimmes, D. (2014). Data acquisition and signal analysis from measured motor currents for defect detection in electromechanical drive systems. European Conference of the Prognostics and Health Management Society, July 8-10, Nantes, France.

Li, H., Ye, X., Chen, C., \& Vachtsevanos, G. (2014). A framework for model-based diagnostics and prognostics of switched-mode power supplies. Annual Conference of the Prognostics and Health Management Society, September 29 - October 2, Fort Worth, TX, USA.

Mohan, N. (2003). First Course on Power Electronics and Drive. Minnesota: MNPERE.

Nandi, S., Toliyat, H.A., \& Li, X. (2005). Condition monitoring and fault diagnosis of electrical motors - a review. IEEE Transactions on Energy Conversion, vol. 20 (4), pp. 719-729.

Nordin, M., Gallic, J., \& Gutman, P.O. (1997). New models for backlash and gear play. International Journal od Adaptive Control and Signal Processing, vol. 11, pp. 49-63.

Orchard, M. (2007). A particle filtering-based framework for on-line fault diagnosis and failure prognosis, Doctoral dissertation, Georgia Institute of Technology, Atlanta, GA, USA.

Orchard, M., \& Vachtsevanos, G. (2009). A particle filtering approach for on-line fault diagnosis and failure prognosis, Transactions of the Institute of Measurement and Control, vol. 31 (3-4), pp. 221-246.

Phillips, W.F. (2004). Mechanics of Flight. Hoboken, NJ, USA: John Wiley \& Sons.

Pratt, R. (2000). Flight control systems: practical issues in design and implementation. UK: IET.

Recksieck, M. (2012). Advanced high lift system architecture with distributed electrical flap. Proceedings of the $2^{\text {nd }}$ International Workshop on Aircraft System Technologies, March 26-27, Hamburg, Germany.

Roemer, M.J., Tang, L.. (2015). Integrated vehicle health and fault contingency management for UAVs In Valavanis, K \& Vachtsevanos, G. Handbook of unmanned aerial vehicle, Berlin, Germany: Springer.

Roemer, M., Byngton, C., Kackprszynski, G., Vachtsevanos, G., \& Goebel, K. (2011). Prognostics In 
Johnson, S.B., System Health Management with Aerospace Applications. Hoboken, NJ: John Wiley \& Sons, pp. 281-295.

Saxena, A., Celaya, J., Balaban, E., Goebel, K., Sasha, B., \& Schwabacher M. (2008). Metrics for evaluating performance of prognostic techniques, International Conference on Prognostics and Health Management, October 6-9, Denver, CO, USA.

Schmidt, M., \& Lipson, H. (2009). Distilling Free-Form Natural Laws from Experimental Data. Science, vol. 324 (5923), pp 81-85.

Vachtsevanos, G., Lewis, F. L., Roemer, M., Hess, A., \& $\mathrm{Wu}, \mathrm{B}$. (2006). Intelligent fault diagnosis and prognosis for engineering system. Hoboken, NJ: John Wiley \& Sons, Inc.

Van Der Linden, F. L.J., Dreyer, N., \& Dorkel, A. (2016). EMA Health Monitoring: An Overview. Recent Advances in Aerospace Actuation Systems and Components, March 16-18, Toulouse, France.

Wang, L., Maré, J.C., \& Fu, Y. (2012). Investigation in the dynamic force equalization of dissimilar redundant actuation systems operating in active/active mode. 28th International Congress of the Aeronautical Sciences, September 23-28, Brisbane, Australia.

\section{BIOGRAPHIES}

Andrea De Martin is currently Ph.D. student at Politecnico di Torino, where he obtained his master degree in mechanical engineering in 2013. His main research interests are the development of novel components for aerospace applications and the study of prognostics and health management solutions for aeronautic systems. He is a member of the Prognostics and Health Management Society.

Giovanni Jacazio is full professor of applied mechanics and of mechanical control systems. His main research activity is in the area of aerospace control and actuation systems and of prognostics and health management. He is a member of the SAE A-6 Committee on Aerospace Actuation Control and Fluid Power Systems, and a member of the International Society of Prognostics and Health Management.

George J. Vachtsevanos is a Professor Emeritus of Electrical and Computer Engineering at the Georgia Institute of Technology and also serves as the chief scientist of Impact Technologies, LLC. He has published over 250 technical papers and is a senior member of IEEE. Dr. Vachtsevanos was awarded the IEEE Control Systems Magazine Outstanding Paper Award for the years 20022003 (with L. Wills and B. Heck). He was also awarded the 2002-2003 Georgia Tech School of Electrical and Computer Engineering Distinguished Professor Award and the 20032004 Georgia Institute of Technology Outstanding Interdisciplinary Activities Award. 